\title{
An Algorithm of Selecting more than Four Satellites from GNSS ${ }^{*}$
}

\author{
Dan Song, Pengfei Zhang, Guochao Fan and Chengdong Xu \\ School of Aerospace Engineering, Beijing Institute of Technology, Beijing, China \\ songdan0207@163.com, successful.2008@163.com
}

\begin{abstract}
By analyzing the relationship between the GDOP value and the area of the polygon formed with the endpoints of the satellite-receiver unit vectors in 2-D, the optimal satellite-receiver geometry (SRG) and the minimum GDOP value of $n$ satellites $(n \geq 4)$ in 3-D were deduced in this paper. Based on the minimum GDOP value and the optimal SRG in 3-D, a satellite selection algorithm is proposed to solve the problem of selecting more than 4 satellites from GNSS (Global Navigation Satellite Systems). The good performance of this algorithm on improving satellite selection efficiency with less sacrifice of the GDOP value is illustrated by simulation.

Index Terms - Satellite selection, GDOP, Satellite-receiver geometry (SRG), GNSS.
\end{abstract}

\section{Introduction}

GDOP (geometric dilution of precision) has been the standard of satellite selection for a long time because it is an important concept to characterize the positioning accuracy. The smaller the GDOP value is, the higher the positioning accuracy will be $[1,2,3,4]$.GDOP is defined as

$$
G D O P=\sqrt{\operatorname{Tr}\left(G^{T} G\right)^{-1}}
$$

Here $G$ is known as the geometry matrix since it characterizes the satellite-receiver geometry (SRG) which is formed with the endpoints of receiver-satellite unit vectors [1] as seen in Fig. 1. $\operatorname{Tr}(\square)$ is the trace of a matrix.

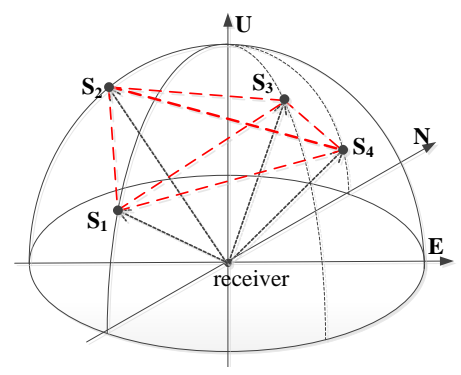

Fig. 1 The SRG of 4 satellites

$$
G=\left[\begin{array}{cccc}
x_{1} & y_{1} & z_{1} & 1 \\
x_{2} & y_{2} & z_{2} & 1 \\
\vdots & \vdots & \vdots & 1 \\
x_{n} & y_{n} & z_{n} & 1
\end{array}\right]
$$

Where $n$ is the number of visible satellites for positioning and $P_{i}=\left[\begin{array}{lll}x_{i} & y_{i} & z_{i}\end{array}\right]^{T}$ is the unit vector from the receiver to the satellite $i(i=1,2, \cdots, n)$.

Positioning with GNSS (Global Navigation Satellite Systems, including GPS, GLONASS, Galileo System and BDS), at least 4 satellites should be selected to solve an equation set with 4 unknown parameters (the receiver position in 3-D and its clock offset).

When the number of satellites is $4, G$ is a square matrix. Formula (1) can be expressed as

$$
G D O P=\sqrt{\operatorname{Trace}\left(G^{*}\left(G^{T}\right)^{*}\right)} /|G|
$$

Where $G^{*}$ is the adjoint matrix of $G$, and $|G|$ is the determinant of $G$. It should be noted that $|G|$ is six times of the volume of the SRG tetrahedron. $\sqrt{\operatorname{Trace}\left(G^{*}\left(G^{T}\right)^{*}\right)}$ changes little when the value of $|G|$ increases. Therefore the GDOP value is almost inversely proportional to the SRG tetrahedron's volume [5]. As a result, the minimum GDOP value of 4 satellites can be obtained when the SRG tetrahedron's volume reaches maximum. Researchers call the SRG minimizing GDOP value the optimal SRG [1, 3, 4, 5]. So the optimal SRG of 4 satellites is the one which owns the largest volume- one satellite at the zenith and the other three in the horizontal plane to constitute an equilateral triangle [6].

For decades, it's common to position with selecting four satellites not only because of the scarce of satellite resources (only 6 to 12 satellites for most regions [6]) but also the almost inverse proportionality relationship between the volumes of tetrahedron and the GDOP values[4,6]. It's easy to select 4 satellites with the largest SRG tetrahedron which is closest to the optimal. However, no matter how effective the satellite selection algorithms are, the positioning accuracy of 4 selected satellites can't be further improved due to the limitation of the satellite number. Moreover, thanks to the development of the receiver manufacturing technology, most receivers can receive signal from more than 12 visible satellites. In addition, with the development of GNSS, the number of all-in-view satellites increases rapidly nowadays. After BDS and Galileo System built, there may be more than 40 visible satellites for most regions on the Earth [2, 7]. The era of satellite resource

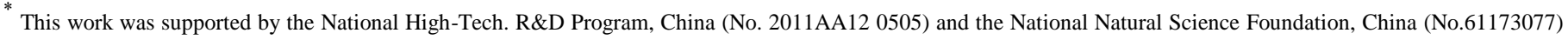
to Prof. Chengdong Xu. 
scarcity has gone. Consequently, selecting more than four satellites from different constellations of GNSS to reduce the GDOP value to improve the positioning accuracy is needed and can be achieved.

However, when the number of selected satellites is more than $4, G$ is no longer a square matrix so that Formula (3) is not established. In addition, the relationship between the volume of the SRG polyhedron and the matrix $G$ is uncertain. So we can't define the optimal SRG as the largest volume one for more than 4 satellites. Because the optimal SRG is not clear, it's hard to obtain the minimum GDOP value of $n$ satellites $(n \geq 4)$ directly. Therefore, the minimum GDOP value and the optimal SRG of $n$ satellites $(n \geq 4)$ are both of theoretical and practical interest. There have been quite a few research efforts [1, 3, 4, 9], for example, by studying the relationship between the shape of polygon and the GDOP value in 2-D, Nuria [1] proved that the SRG close to a regular polygon is optimal to minimize the GDOP value. However, this theory is only applicable to 2-D. Miaoyan [3] summarized formulas for calculating the minimum GDOP value of $n$ satellites $(n \geq 4)$ for both 2 -D and 3-D positioning. But the derivation should be more detailed.

If the optimal SRG and minimum GDOP value of $n$ satellites $(n \geq 4)$ was found in $3-\mathrm{D}$, the satellite selection algorithm for selecting more than 4 satellites from GNSS could be designed to decreasing the GDOP value. There are many studies on algorithm of selecting 4 satellites [10], but the research of satellite selection beyond 4 satellites is infrequent. Miaoyan [7] designed a fast satellite selection algorithm for more than 4 satellites which has good performance on decreasing the GDOP value. However, the method of grouping visible satellites in that algorithm is not comprehensive. Nuria [11] proposed a satellite selection method for GNSS using convex geometry, but it is limited to 2-D satellite selection.

This paper is organized as follows. In Section 2 and 3, starting with analyzing the relationship between the GDOP and the area of the SRG polygon formed in 2-D, the optimal SRG of $n(n \geq 4)$ satellites in $3-\mathrm{D}$ is deduced by calculating the minimum GDOP value with a formula which is speculated and verified by simulation. In Section 4, an algorithm of selecting more than 4 satellites from GNSS (ASMS) in 3-D is proposed. And two simulation experiments are designed to illustrate the effectiveness of ASMS on decreasing the GDOP value for selecting $n$ satellites $(n \geq 4)$. Section 5 is the conclusion.

\section{II . The minimum GDOP value and the SRG for more than four satellites in 2-D}

There is a direct relationship between the GDOP value and the area of the SRG polygon defined in 2-D. The GDOP value decreases with the increase of the SRG polygon's area. The minimum GDOP value appears when the area of the polygon is largest [1].

Because the SRG polygon is a unit circle inscribed polygon, its area will be the largest when it is a regular polygon. So the optimal SRG for $n$ satellites is the regular polygon in 2-D, the minimum GDOP value can be calculated as follows:

The geometry matrix $G$ in 2-D is

$$
G=\left[\begin{array}{ccc}
x_{1} & y_{1} & 1 \\
x_{2} & y_{2} & 1 \\
\vdots & \vdots & \vdots \\
x_{n} & y_{n} & 1
\end{array}\right]
$$

Where $n$ is the number of visible satellites selected for positioning and $P_{i}=\left[\begin{array}{ll}x_{i} & y_{i}\end{array}\right]^{T}$ is the unit vector from the receiver to the satellite $i(i=1,2, \cdots, n)$. Thus

$$
G^{T} G=\left[\begin{array}{ccc}
\sum_{i=1}^{n} x_{i}^{2} & \sum_{i=1}^{n} x_{i} y_{i} & \sum_{i=1}^{n} x_{i} \\
\sum_{i=1}^{n} x_{i} y_{i} & \sum_{i=1}^{n} y_{i}^{2} & \sum_{i=1}^{n} y_{i} \\
\sum_{i=1}^{n} x_{i} & \sum_{i=1}^{n} y_{i} & n
\end{array}\right]
$$

So $\operatorname{Tr}\left(G^{T} G\right)=\sum_{i=1}^{n}\left(x_{i}^{2}+y_{i}^{2}\right)+n=2 n$ because $x_{i}^{2}+y_{i}^{2}=1$.

Assume that the eigenvalues of $G^{T} G$ are $\lambda_{1}, \lambda_{2}$ and $\lambda_{3}$, thus $\operatorname{Tr}\left(G^{T} G\right)=\lambda_{1}+\lambda_{2}+\lambda_{3}=2 n$. Therefore the eigenvalues of $\left(G^{T} G\right)^{-1}$ are $1 / \lambda_{1}, 1 / \lambda_{2}$ and $1 / \lambda_{3}$ obviously. So the GDOP value is

$$
G D O P=\sqrt{\operatorname{Tr}\left(G^{T} G\right)^{-1}}=\sqrt{\left(1 / \lambda_{1}\right)+\left(1 / \lambda_{2}\right)+\left(1 / \lambda_{3}\right)}
$$

When the polygon is a regular one, $\sum_{i=1}^{n} x_{i}=\sum_{i=1}^{n} y_{i}=\sum_{i=1}^{n} x_{i} y_{i}=0$ and $\sum_{i=1}^{n} x_{i}^{2}=\sum_{i=1}^{n} y_{i}^{2}=\frac{n}{2}$. Formula (5) can be simplified as

$$
G^{T} G=\left[\begin{array}{ccc}
0.5 n & 0 & 0 \\
0 & 0.5 n & 0 \\
0 & 0 & n
\end{array}\right]
$$

Thus $\lambda_{1}=\lambda_{2}=0.5 n, \lambda_{3}=n$.Substitute them into the Formula (6), the minimum GDOP value of $2-\mathrm{D}$ can be denoted as

$$
G D O P=\sqrt{5 / n}
$$

Because the optimal SRG is the regular polygon, selecting the satellites whose SRG approximates the regular polygon is helpful to improve positioning accurately by decreasing the GDOP in 2-D. However, in actual situation, the satellite position is described in 3-D. It's necessary to find the optimal 3-D SRG of $n$ satellites $(n \geq 4)$ to minimize the GDOP value. Inspired from the derivation of 2-D optimal SRG and the minimum GDOP value, we can start with the matrix $G^{T} G$ to surmise the minimum GDOP value in 3-D. Thereby the 3-D optimal SRG can be found.

\section{The minimum GDOP value and the USG for more than four satellites in 3-D}

The geometry matrix $G$ in 3-D is shown as Formula (2), 
thus $G^{T} G$ is expressed as

$$
G^{T} G=\left[\begin{array}{cccc}
\sum_{i=1}^{n} x_{i}^{2} & \sum_{i=1}^{n} x_{i} y_{i} & \sum_{i=1}^{n} x_{i} z_{i} & \sum_{i=1}^{n} x_{i} \\
\sum_{i=1}^{n} x_{i} y_{i} & \sum_{i=1}^{n} y_{i}^{2} & \sum_{i=1}^{n} y_{i} z_{i} & \sum_{i=1}^{n} y_{i} \\
\sum_{i=1}^{n} x_{i} z_{i} & \sum_{i=1}^{n} y_{i} z_{i} & \sum_{i=1}^{n} z_{i}^{2} & \sum_{i=1}^{n} z_{i} \\
\sum_{i=1}^{n} x_{i} & \sum_{i=1}^{n} y_{i} & \sum_{i=1}^{n} z_{i} & n
\end{array}\right]
$$

In Formula (5), All of non-diagonal elements of $G^{T} G$ are zero element when the GDOP value is minimum in 2-D. It can be supposed that in Formula (9), the matrix $G^{T} G$ may be diagonal matrix or similar to the diagonal matrix when the GDOP value is minimum. According to the derivation from Formula (5) to Formula (7), to make $G^{T} G$ similar to a diagonal matrix, some of the satellites should be in the horizontal plane and constitute a regular polygon; others should be at the zenith. Thus the matrix $G$ expresses as

$$
G=\left[\begin{array}{cccc}
0 & 0 & 1 & 1 \\
\vdots & \vdots & \vdots & \vdots \\
0 & 0 & 1 & 1 \\
x_{m+1} & y_{m+1} & 0 & 1 \\
x_{m+2} & y_{m+2} & 0 & 1 \\
\vdots & \vdots & \vdots & \vdots \\
x_{n} & y_{n} & 0 & 1
\end{array}\right]
$$

Where $m$ is the number of satellites at the zenith, $n$ is the total number of visible satellites for positioning. Substitute Formula (10) into Formula (9), it can be obtained that

$$
G^{T} G=\left[\begin{array}{cccc}
\sum_{i=m+1}^{n} x_{i}^{2} & \sum_{i=m+l}^{n} x_{i} y_{i} & 0 & \sum_{i=m+1}^{n} x_{i} \\
\sum_{i=m+1}^{n} x_{i} y_{i} & \sum_{i=1}^{n} y_{i}^{2} & 0 & \sum_{i=m+1}^{n} y_{i} \\
0 & 0 & m & m \\
\sum_{i=m+l}^{n} x_{i} & \sum_{i=m+1}^{n} y_{i} & m & n
\end{array}\right]
$$

Because the satellites from $m+1$ to $n$ constitute a regular polygon, refer to Formula (7), Formula (11) can be simplified as

$$
G^{T} G=\left[\begin{array}{cccc}
0.5(n-m) & 0 & 0 & 0 \\
0 & 0.5(n-m) & 0 & 0 \\
0 & 0 & m & m \\
0 & 0 & m & n
\end{array}\right]
$$

Assume that the eigenvalues of $G^{T} G$ in Formula (12) are $\lambda_{1}, \lambda_{2}, \lambda_{3}$ and $\lambda_{4}$, thus $\operatorname{trace}\left(G^{T} G\right)=\lambda_{1}+\lambda_{2}+\lambda_{3}+\lambda_{4}=2 n$, $\lambda_{1}=\lambda_{2}=(n-m) / 2, \lambda_{3}=m(n-m) / n, \lambda_{4}=n-m$. The eigenvalues of $\left(G^{T} G\right)^{-1}$ are $1 / \lambda_{1}, 1 / \lambda_{2}, 1 / \lambda_{3}$ and $1 / \lambda_{4}$. So according to Formula(1) and Formula(6), the GDOP value can be recorded as

$$
G D O P=\sqrt{(5 m+n) /[m(n-m)]}
$$

Obviously, to minimize the GDOP value, there should be different combinations of $n$ and $m$ satisfying:

$$
5 m^{2}+2 m n-n^{2}=0
$$

TABLE I shows the different values of $m$ (the numbers of satellites at the zenith) for the total satellites number $n$ changes from 4 to 12 in the second column.

According to Formula (13), the minimum GDOP values for different $n$ can be calculated (Table I, the third column). However, as Formula (13) is built on assumption, a simulation is needed to test and verify its reliability. Without considering the actual situation of the constellation distribution of GNSS, in simulation, the receiver-satellite unit vectors are randomly generated. For different value of $n$ (the number of visible satellites for positioning), GDOP value is calculated for $10^{6}$ times respectively. The simulation results are shown in the fourth column of TABLE I.

TABLE I The number of satellites at the zenith and the minimum GDOP values of $n$ satellites

\begin{tabular}{|c|c|c|c|}
\hline $\begin{array}{c}n \\
\text { (Total } \\
\text { number of } \\
\text { satellites) }\end{array}$ & $\begin{array}{c}m \\
\text { (The number of } \\
\text { satellites at } \\
\text { zenith) }\end{array}$ & $\begin{array}{c}\text { Theoretical } \\
\text { minimum } \\
\text { GDOP values }\end{array}$ & $\begin{array}{c}\text { Experimental } \\
\text { Minimum GDOP } \\
\text { values }\end{array}$ \\
\hline 4 & 1 & 1.7321 & 1.7639 \\
\hline 5 & 1 & 1.5811 & 1.6144 \\
\hline 6 & $1(2)$ & 1.4142 & 1.4125 \\
\hline 7 & 2 & 1.3038 & 1.3811 \\
\hline 8 & 2 & 1.2247 & 1.2941 \\
\hline 9 & 3 & 1.1547 & 1.2221 \\
\hline 10 & 3 & 1.0911 & 1.1701 \\
\hline 11 & 3 & 1.0408 & 1.1086 \\
\hline 12 & 3 & 1.0000 & 1.0938 \\
\hline
\end{tabular}

Comparing the theoretical minimum GDOP values with the experimental ones in TABLE I, Formula (13) is certified to be effective. Meanwhile, the optimal SRG of $n$ satellites for positioning in 3-D is that $m$ satellites are at the zenith and others are in the horizontal plane to constitute a regular polygon. The value $m$ can be searched in Table 1 or calculated according to Formula (14).

\section{An algorithm of selecting more than four satellites from GNSS (ASMS)}

Among all-in-view satellites, the closer the SRG of the selected ones is to the optimal SRG, the smaller the GDOP value will be. So ASMS is devised to select $n$ satellites $(n \geq 4)$ which can constitute the SRG similar to the optimal SRG mentioned in Section 3. Assume the total number of selected satellites is $n$. The steps of this algorithm are as follows:

Step 1 Compare the number of all-in-view satellites $(N)$ and selected satellites $(n)$. If $N<=n$, all of visible satellites should be selected; else go Step 2. 
Step 2 Calculate the zenith satellites number $m$ with Formula (14) according to the total selected satellites number $n$. And Select the first $m$ visible satellites from all-in-view satellites according to their elevation angles which are listed in descending order.

Step 3 Find out the visible satellite from all-in-view satellites which has the minimum elevation angle and denote it as A.

Step 4 Divide all-in-view satellites into $n-m$ groups except the ones selected in Step 2 and Step 3.

The azimuths of satellites in the Group $j$ should satisfy the inequality

$$
a z i_{A}+\frac{360(j-0.5)}{n-m}<a z i \leq a z i_{A}+\frac{360(j+0.5)}{n-m}
$$

Where $a z i_{A}$ is the azimuth of satellite A and $a z i$ denotes the azimuth of a visible satellite in the Group $j(j=1,2, \cdots, n-m)$.

The grouping can be shown in Fig. 2 which describes the orientation relationship between the receiver and all-in-view satellites in topocentric coordinate system. $\boldsymbol{O}$ is the position of the receiver. The farther the dot from $\boldsymbol{O}$ is, the lower the elevation angle of the satellite denoted by the dot will be. Circle $\boldsymbol{C}$ means horizon orientation. The yellow hexagons denote the zenith satellites selected in Step2.The green fivepointed star denotes satellite A selected in Step3. The red triangles are drawn according to the orientation of satellite A. They represent the virtual satellites which can constitute the optimal SRG with the visible satellite selected in Step 2 and Step 3. The purple dots denote the grouped satellites.

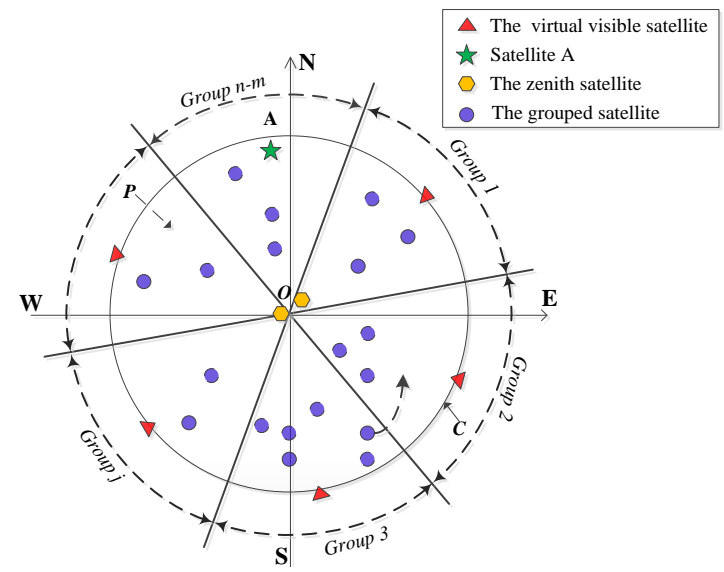

Fig. 2 Satellite selection process

\section{Step 5 Adjust the grouping.}

Sometimes, all the elevation angles of visible satellites in Group $j$ are large enough to exceed a threshold (maybe $30^{\circ}$ ), just like Group 2 in Fig. 3. If one of them is selected, the polygon composed by the selected satellites except the ones at the zenith may be a concave polygon in topocentric coordinate system. Thus the satellite selection will be disappointing. Therefore, the grouping should be adjusted.

The group adjustment process can be seen in Fig. 3 .

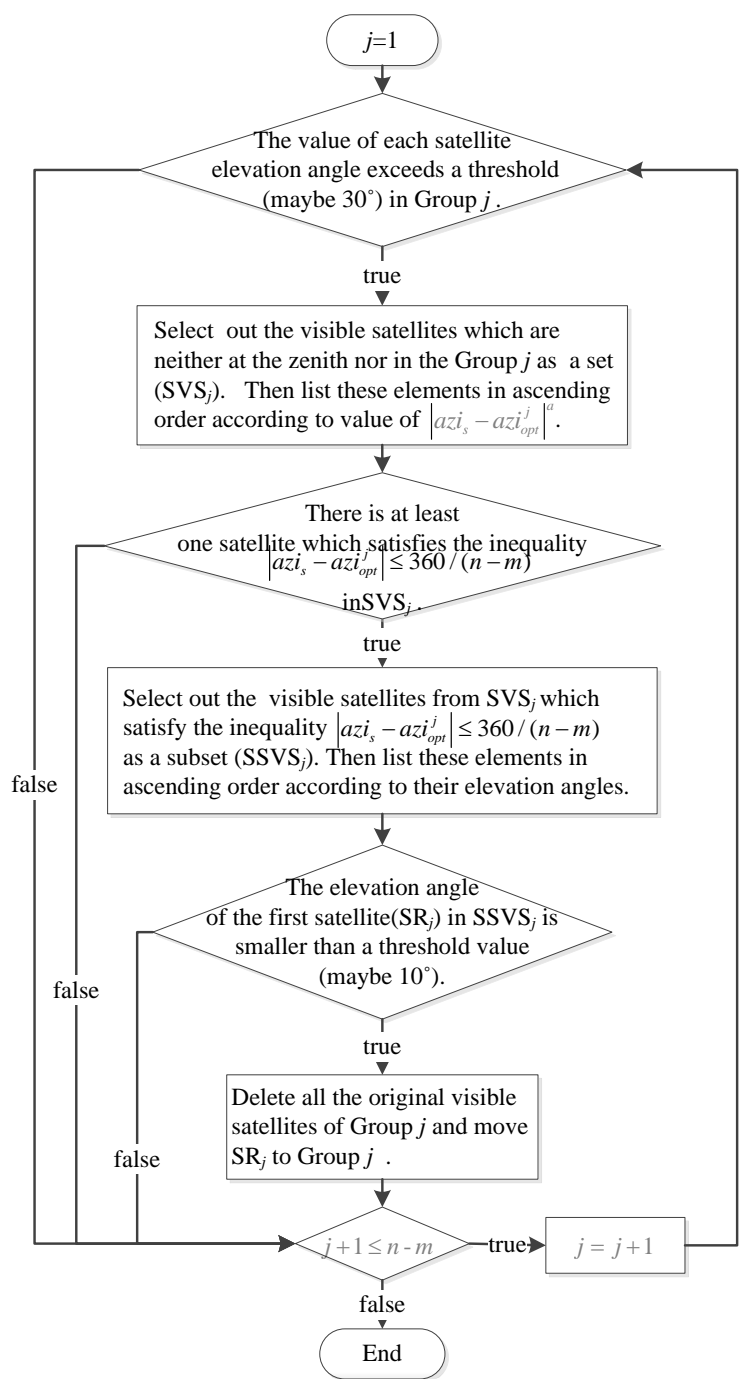

Fig. 3 The group adjustment process

${ }^{a} a z i_{s}$ denotes the azimuth of the visible satellite. $s=1,2, \cdots, n-m-1-l_{j}, l_{j}$ is the total number of visible satellites in Group $j . a z i_{\text {opt }}^{j}=360 j /(n-m)+a z i_{A}$.

Step 6 Select out one satellite in each group except the Group $n-m$ and combine them with the satellites selected in Step 3 and Step 4 as a subset. Thus there will be $l_{1} \times l_{2} \times \cdots \times l_{n-m-1}$ different subsets, where $l_{j}$ is the total satellites number of Group $j(j=1,2, \cdots, n-m-1)$.Traverse all the subsets and find out the one minimize the GDOP value. This subset is the satellite selection result.

To illustrate the validity of ASMS, two simulation experiments are designed. In both experiments, the GDOP value of the point $S$ at North latitude $39^{\circ}$, East longitude $116^{\circ}$ is calculated from 2011-1-1 5:00 to 2011-1-1 7:00 with the sampling interval of $2 \mathrm{~min}$. The satellites are selected from GPS, GLONASS and Galileo. The number of all-in-view satellites is from 31 to 35 under this default scene.

In the first experiment, each GDOP value of selected 8 satellites using ASMS is compared with the minimum one among all the GDOP values of optionally random selecting 8 
satellites for $1 \times 10^{6}$ times. The simulation result can be seen in Fig. 4 and TABLE II.

Analysing the statistical data in TABLE II, it's undeniable that the satellite selection result of ASMS is not the optimal. However there will be about ten million random combinations of different 8 satellites $\left(C_{35}^{8}\right)$. It is impossible to traverse all the combinations and find the one which minimize the GDOP value for its huge amount of calculation and time-consuming (tens of minutes for once). ASMS can finish the calculation process within less than 100 milliseconds. Under the premise of sacrificing the GDOP value a little, it reduces the computation and improves the satellite selection speed.

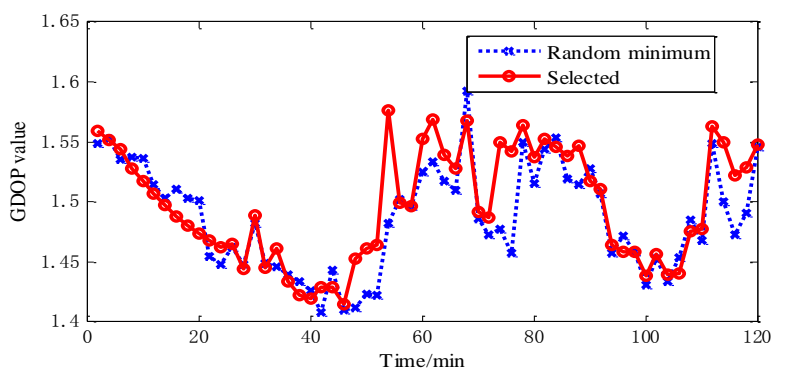

Fig. 4 GDOP value for selected 8 satellites and random 8 satellites in the first simulation experiment

TABLE II The statistical data of the first experiment

\begin{tabular}{|c|c|c|c|}
\hline The GDOP value & Mean & Variance & Standard deviation \\
\hline Selected 8satellites & 1.4973 & 0.0022 & 0.0467 \\
\hline Random 8 satellites & 1.4780 & 0.0020 & 0.0443 \\
\hline
\end{tabular}

In the second experiment, ASMS is used to respectively select 10, 14, 18 and 22 satellites from GPS and Galileo. The simulation result is shown in Fig. 5.

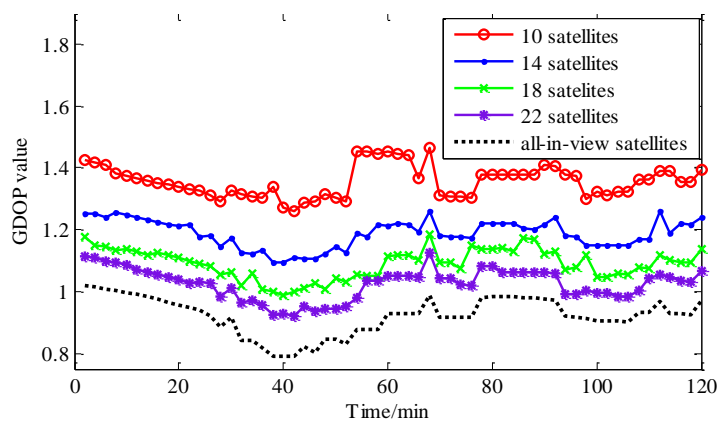

Fig. 5 GDOP value for different number of selected satellites

As seen in Fig. 5, with the increasing of the number of satellites selected by ASMS, the GDOP value decreases. The GDOP value is close to that of all-in-view satellites with the increasing of selected satellites number.

It can be confirmed from above two experiments that although the satellite selection may not be the optimal, compared with traversing all of satellites combinations, ASMS greatly reduces computation. Moreover, with the increasing of selected satellites number, the GDOP value decreases. Therefore it can be studied determining the number of selected satellites according to the receiver's needs of positioning accuracy.

\section{Conclusions}

Starting with derivation the relationship of the optimal SRG and the minimum GDOP value in 2-D, a rule to obtain the minimum GDOP value has been found, which is to make matrix $G^{T} G$ a diagonal matrix. Through this rule, a formula (Formula (13)) that is used to calculate the minimum GDOP value of $n$ satellites in 3-D was deduced and its reliability was verified by simulation. Meanwhile the optimal SRG to minimize the GDOP value in 3-D was summarized: for $n$ satellites $(n \geq 4)$, there should be $m$ at the zenith and $m-n$ in the horizontal plane to constitute a regular polygon.

After analysing the minimum GDOP value and the optimal SRG of $n$ satellites in 3-D, an algorithm of selecting more than 4 satellites from GNSS (ASMS) was proposed. ASMS can get the small GDOP value and improve satellite selection efficiency by selecting the satellites whose SRG is close to the optimal one. By two simulation experiments, the good performance of ASMS is illustrated.

Because the thresholds of elevation angle in Step 5 are to be determined, ASMS should be studied further.

\section{References}

[1] Nuria Blanco-Delgado, Fernando D. Nunes and Gonzalo Seco-Granados, "Relation between GDOP and the Geometry of the Satellite Constellation", 2011 International Conference on Localization and GNSS,USA, pp.75-180,June 1987.

[2] Lu Yun and Yang Qiangwen, "Study on GNSS Interoperability". SCIENTIA SINICA Phys, Mech \& Astron, vol.40, no. 9, pp. 534-540, 2010.

[3] Miaoyan Zhang and Jun Zhang, "A Fast Satellite Selection Algorithm: Beyond Four Satellites", Journal of Selected Topics in Signal Processing,vol.30, no. 5,pp. 740-747, 2009.

[4] Mok E. and Cross P.A., "A fast satellite selection algorithm for combined GPS and GLONASS receivers", Journal of Navigation, vol. 40, pp. 283$389,1994$.

[5] CONG Li, Ahmed I Abidat and TAN Zhan-zhong, "Analysis and Simulation of the GDOP of Satellite Navigation", ACTA ELECTRONICA SINICA, vol. 34, no. 9,pp. 2204-2208, 2006.

[6] Hofman-Wellenhof, Lichtenegger and Wasle, GNSS-Global Navigation Satellite Systems GPS, GLONASS, Galileo \& more, Spring-Verlag, 2008, pp. 197-199.

[7] Zhang Miaoyan, Zhang Jun and Qin Yong, Satellite Selection for MultiConstellation, 2008 IEEE/ION Position, Location and Navigation Symposium, USA, pp. 1053-1059, Monterey, May 2008.

[8] ZHOU ShanShi, et al, "Positioning accuracy assessment for the 4GEO/5IGSO/2MEO constellation of COMPASS", SCIENCE CHINA Physics, Mechanics \& Astronomy, vol.55, No.12, pp. 2290-2299, 2012.

[9] Massatt P. and Rudnick K., "Geometric Formulas for Dilution of Precision Calculations", Journal of the Institute of Navigation, vol.37, no. 4, pp. 379-391,1991.

[10]ZHENG Zuoya, et al, "Selection of GPS Satellites for the Optimum Geometry", Chinese Astronomy and Astrophysics, vol.28, pp. 80-87, 2003.

[11]Nuria Blanco-Delgado and Fernando D. Nunes, "Satellite Selection Method for Multi-Constellation GNSS Using Convex Geometry", IEEE Translated. Vehicular Technology, USA, vol.59, pp. 4289-4297, 2010. 